\title{
Doenças mentais relacionadas ao trabalho: um levantamento sobre a saúde da equipe de enfermagem
}

\author{
Mental diseases related to work: a survey on the health of the nursing team
}

Enfermedades mentales relacionadas con el trabajo: una encuesta sobre la salud del equipo de enfermería

\section{Anna Carolina Siqueira Veneu ${ }^{1}$, Jorge Luiz Lima da Silva ${ }^{2 *}$, João Victor Lima da Silva ${ }^{3}$, Cristhian Antonio Brezolin ${ }^{4}$, Gilvania Barreto Feitosa Coutinho ${ }^{5}$, Vinicius Fonseca de Lima ${ }^{6}$}

Como citar esse artigo. Veneu, ACS; da Silva, JLL; da Silva, JVL; Brezolin, CA; Coutinnho, GBF; de Lima, VF. Doenças mentais relacionadas ao trabalho: um levantamento sobre a saúde da equipe de enfermagem Revista Pró-UniverSUS. 2020 Jul./Dez.; 11 (2): 102-110.

\section{Resumo}

Introdução: o perfil do trabalho e dos trabalhadores modificou-se ao longo dos anos para se adaptar a introdução de novas tecnologias e, juntamente com isso, houve intensificação do trabalho que foram determinantes do processo saúde-doença desses trabalhadores. Objetivo: descrever as principais doenças relacionadas ao trabalho que afetam a equipe de enfermagem no Brasil. Materiais e Métodos: trata-se de uma revisão integrativa, a busca foi realizada por meio de levantamento de periódicos nacionais nas bases de dados Lilacs (Literatura Latino-Americana, do Caribe em Ciências da Saúde) e Bdenf (Base de dados de Enfermagem), Medline (Medical Literature Analysis and Retrieval System Online) e nas bibliotecas virtuais Scientific Eletronic Library Online, respeitando o limite temporal de 2010-2020. Resultados: a amostra foi composta de 12 artigos. Foi constatado que é necessário enfrentar o desafio de compreender os fenômenos em saúde mental e de lidar com situações complexas, dadas as múltiplas manifestações do sofrimento relacionado ao trabalho, suas diferenças e níveis. Conclusão: o quadro de adoecimento coletivo de enfermagem é alarmante, por conseguinte urge modificá-lo através de estudos que busquem medidas de prevenção, enfrentamento dos agravos e riscos à saúde dos trabalhadores da equipe de enfermagem.

Palavras-chave: Saúde Do Trabalhador; Transtornos Mentais; Absenteísmo; Equipe de Enfermagem.

\begin{abstract}
Introduction: the profile of work and workers has changed over the years to adapt to the introduction of new technologies and together with this, there has been an intensification of work that were determinants of the health-disease process of these workers. Objective: to describe the main work-related diseases that affect the nursing team in Brazil. Materials and Methods: this is an integrative review, the search was carried out by surveying national journals in the Lilacs (Latin American, Caribbean Literature in Health Sciences) and Bdenf (Nursing Database) databases, Medline (Medical Literature Analysis and Retrieval System Online) and the Scientific Electronic Electronic Library Online virtual libraries, respecting the 2010-2020 time limit. Results: the sample consisted of 12 articles. It was found that it is necessary to face the challenge of understanding the phenomena in mental health and dealing with complex situations, given the multiple manifestations of suffering related to work and its differences and levels. Conclusion: the picture of collective illness of nursing is alarming, therefore it is urgent to modify it, through studies that seek measures to prevent and cope with the health problems and risks of the workers of the nursing team. Keywords: Occupational Health; Mental Disorders; Absenteeism; Nursing Team.
\end{abstract}

\section{Resumen}

Introducción: el perfil del trabajo y los trabajadores ha cambiado a lo largo de los años para adaptarse a la introducción de nuevas tecnologías y, junto con esto, ha habido una intensificación del trabajo que fue determinante del proceso de salud-enfermedad de estos trabajadores. Objetivo: describir las principales enfermedades relacionadas con el trabajo que afectan al equipo de enfermería en Brasil. Materiales y métodos: esta es una revisión integradora, la búsqueda se realizó mediante encuestas en revistas nacionales en las bases de datos Lilacs (Literatura Latinoamericana, del Caribe en Ciencias de la Salud) y Bdenf (Base de Datos de Enfermería), Medline (Sistema de recuperación y análisis de literatura médica en línea) y las bibliotecas virtuales en línea de la Biblioteca Electrónica Científica y Electrónica, respetando el límite de tiempo 2010-2020. Resultados: la muestra consistió en 12 artículos. Se descubrió que es necesario enfrentar el desafío de comprender los fenómenos de la salud mental y enfrentar situaciones complejas, dadas las múltiples manifestaciones del sufrimiento relacionado con el trabajo y sus diferencias y niveles. Conclusión: la imagen de la enfermedad colectiva de la enfermería es alarmante, por lo tanto, es urgente modificarla, a través de estudios que buscan medidas para prevenir y hacer frente a los problemas y riesgos de salud de los trabajadores del equipo de enfermería.

Palabras clave: Salud Del Trabajador; Desordenes Mentales; Absentismo; Equipo de Enfermería.

Afiliação dos autores:

${ }^{1}$ Universidade Federal Fluminense/Niterói/ Rio de Janeiro. Brasil. ORCID: https://orcid.org/0000-0002-7918-1988

${ }^{2}$ Docente. Universidade Federal Fluminense/Niterói/ Rio de Janeiro. Brasil. ORCID: https://orcid.org/0000-0002-2370-6343

${ }^{3}$ Universidade Federal Fluminense/Niterói/ Rio de Janeiro. Brasil. ORCID: https://orcid.org/0000-0002-5561-0303

${ }^{4}$ Universidade Federal Fluminense/Niterói/ Rio de Janeiro. Brasil. ORCID: https://orcid.org/0000-0002-9525-2459

${ }^{5}$ Fundação Instituto Oswaldo Cruz - Fiocruz - Rio de Janeiro/RJ. Brasil. ORCID: https://orcid.org/0000-0002-9655-4010

${ }^{6}$ Universidade Federal Fluminense/Niterói/ Rio de Janeiro. Brasil. ORCID: https://orcid.org/0000-0002-3301-4105 


\section{Introdução}

O presente trabalho consiste em uma revisão bibliográfica com tema na área de saúde do trabalhador, abordando as doenças mentais que mais acometem a equipe de enfermagem no Brasil. As atuais transformações que estão ocorrendo em todo o mundo propiciam o entendimento de que não podemos mais concordar com o conceito de que o trabalho seja um processo de "robotização" promovendo a "desumanização" do trabalhador e que esteja inserido em qualquer ambiente laboral. Se o homem é roubado no seu próprio trabalho, é roubado de si mesmo, perdese quando deveria se identificar, desconhece a si mesmo quando deveria se conhecer, destrói-se quando deveria estar se construindo ${ }^{1}$.

Os transtornos mentais e comportamentais relacionados ao trabalho são aqueles resultantes de situações que envolvem o processo ou a organização do trabalho, tais como a divisão de tarefas, as políticas de gerenciamento das pessoas, o assédio moral no trabalho e a estruturação hierárquica organizacional conforme a ficha de transtorno mental relacionado ao trabalho do SINAN - Sistema de Informação de Agravos e Notificação.

Atualmente, com base nos dados do Anuário Estatístico da Previdência Social, em 2011 os transtornos mentais foram a causa da terceira razão de afastamento no Brasil, sendo que os gastos do INSS (Instituto Nacional do Seguro Social) giram em torno de R $\$ 200$ milhões (duzentos milhões de reais) em benefícios anuais, dado que reforça a importância de se estudar medidas de prevenção e enfrentamento frente a este problema $^{2}$.

Os profissionais de enfermagem, frequentemente avaliados pelo seu desempenho e exigidos em sua maioria ao máximo, em diversos momentos de sua jornada profissional, torna-se primordial considerar que essas situações podem levar a tensão, e frequentemente, ao sofrimento e a insatisfação do trabalhador, o que pode desencadear, na maioria das vezes, os distúrbios mentais.

As ações implicadas no ato de trabalhar podem atingir o corpo dos trabalhadores, produzindo disfunções e lesões biológicas, mas também reações psíquicas às situações de trabalho patogênicas, além de poderem desencadear processos psicopatológicos especificamente relacionados às condições do trabalho desempenhado pelo trabalhador ${ }^{3}$.

A Saúde do Trabalhador constitui uma área da Saúde Pública que tem como objeto de estudo a intervenção das relações entre o trabalho e a saúde. Tem como objetivos a promoção e a proteção da saúde do trabalhador, por meio do desenvolvimento de ações de vigilância dos riscos presentes nos ambientes e condições de trabalho, dos agravos à saúde do trabalhador e da organização e prestação da assistência aos trabalhadores, compreendendo procedimentos de diagnóstico, tratamento e reabilitação de forma integrada, no Sistema Único de Saúde (SUS)33.

O trabalho ocupa lugar fundamental na dinâmica do investimento afetivo das pessoas. Condições favoráveis à livre utilização das habilidades dos trabalhadores, da inteligência da prática e que possibilitem aos trabalhadores nível significativo de controle sobre seu processo de trabalho tem sido identificado como importantes requisitos para que a atividade de trabalho possa proporcionar prazer, bemestar e saúde, deixando de provocar doenças ${ }^{4}$. Por outro lado, o trabalho desprovido de significação, sem suporte social, não reconhecido ou que se constitua sem fonte de ameaça à integridade física e/ ou psíquica, pode desencadear sofrimento psíquico.

Objetivo: Descrever as principais doenças mentais relacionadas ao trabalho que afetam a equipe de enfermagem no Brasil.

Justificativa: o interesse em estudar e compreender as doenças mentais que acometem a equipe de enfermagem emergiu ao ingressar na pós-graduação, e floresceu durante as primeiras aulas ministradas em que diversos temas foram abordados, dentre os quais as doenças relacionadas ao trabalho. Ao buscar um aprofundamento dessa temática pode-se observar como os profissionais de saúde, estão diretamente ligados ao surgimento desses transtornos frente à rotina de trabalho.

Opresentetrabalhobuscadestacaraimportânciade se cuidar da saúde mental e ocupacional do profissional, instigando investigações sobre a segurança no trabalho, e vigilância dos agravos à saúde no ambiente de trabalho. As pesquisas dessa natureza expõem características importantes que podem subsidiar estratégias que minimizam o sofrimento dos trabalhadores, e para que se discutam aspectos da organização das tarefas e ambientes laborais 5 .

\section{Materiais e Métodos}

A pesquisa de natureza descritiva foi realizada por meio de revisão bibliográfica sistematizada, baseada em obras secundárias que abordam o tema em questão, publicadas no período de 2010 a 2020. A coleta do material para a pesquisa foi realizada no período entre março e abril de 2020.

O levantamento foi realizado em ambiente virtual na Biblioteca Virtual de Saúde (BVS), nas bases: Lilacs, Bdenf, Medline e Scielo e, em busca livre, de textos completos incluídos, nos resultados com os seguintes termos de busca: "saúde do trabalhador"; "transtornos mentais"; "equipe de enfermagem". Esses termos 
foram utilizados de forma conjunta e isolados. As obras idênticas, repetidas em bases diferentes, foram eliminadas, considerou-se seu primeiro registro.

Foram selecionados para este estudo somente artigos que, na leitura demonstrassem relação com doenças mentais relacionadas ao trabalho, na equipe de enfermagem, utilizando como fonte periódicos da área publicada no Brasil, que estavam disponíveis para leitura de forma completa, conforme descritos na tabela 1. Primeiramente, as obras foram armazenadas em computador, para que, em seguida, fosse realizada préseleção de acordo com a leitura dos resumos. Nessa fase, buscou-se a relação entre o conteúdo, título, resumo, e se atendiam ao objeto do presente estudo.

$\mathrm{Na}$ fase de seleção, as obras foram lidas na íntegra, com atenção especial para os resultados e conclusão das obras, os trabalhos que não apresentavam qualquer relação com transtornos mentais relacionados ao trabalho foram excluídos.

Tendo em vista que o objeto da pesquisa foi estudar as doenças mentais relacionadas ao trabalho entre profissionais de enfermagem, no Brasil. Ao utilizar os termos de busca: "saúde do trabalhador"; "transtornos mentais" e "equipe de enfermagem" foram encontrados 36 artigos, 12 se adequavam aos parâmetros estabelecidos, foram descartados 24 artigos, por não caracterizarem os sujeitos estudados de acordo com o objetivo.

Realizada a triagem das obras foram obtidos 12 artigos, 2 livros para embasamento teórico, 1 arquivos de outras categorias (1 manual). Depois das etapas descritas, foram construídos nos resultados itens que abordam as principais doenças mentais que acometem os enfermeiros no Brasil.

\section{Resultados}

A distribuição dos artigos nos periódicos estudados no período delimitado pode ser analisada conforme destacado na tabela 1 onde se observou que entre 2015 e 2018, foi o período em que houve maior número de publicação sobre esta temática, totalizando 09 (nove) artigos.

Foram encontrados 36 artigos e a amostra final foi composta de 12 artigos (Quadro 1). Destes, 5 foram provenientes da base Scielo, 5 da Lilacs e 2 da BDenf.

A síntese contendo os objetivos, características,

Tabela 1. Distribuição dos artigos de acordo com os periódicos selecionados em ambiente da BVS no período de 2010 a 2020

\begin{tabular}{|c|c|c|c|c|c|c|c|c|c|c|c|c|}
\hline \multirow{2}{*}{ PERIÓDICOS } & \multicolumn{12}{|c|}{ ANO DE PUBLICAÇÃO } \\
\hline & 2010 & 2011 & 2012 & 2013 & 2014 & 2015 & 2016 & 2017 & 2018 & 2019 & 2020 & TOTAL \\
\hline $\begin{array}{l}\text { Rev. Bras. Ter. In- } \\
\text { tensiva }\end{array}$ & - & - & - & - & - & 1 & - & - & - & - & - & 1 \\
\hline Rev. Saúde Pública & - & - & - & - & - & 1 & - & - & - & - & - & 1 \\
\hline Rev. UERJ & - & - & - & - & - & - & - & - & 1 & - & - & 1 \\
\hline $\begin{array}{l}\text { Rev. Cad. Saúde } \\
\text { Pública }\end{array}$ & - & - & - & - & - & 1 & - & - & - & - & - & 1 \\
\hline $\begin{array}{l}\text { Rev. Bras. Ciênc. } \\
\text { Saúde }\end{array}$ & - & - & - & - & - & - & - & - & - & 1 & - & 1 \\
\hline $\begin{array}{l}\text { Rev.Bras. Saúde } \\
\text { Ocup. }\end{array}$ & 1 & - & - & - & - & - & - & - & - & - & - & 1 \\
\hline $\begin{array}{l}\text { Rev. Gaúcha. En- } \\
\text { ferm. }\end{array}$ & - & - & - & - & - & - & 1 & - & - & - & - & 1 \\
\hline $\begin{array}{l}\text { Rev. Bras. Epide- } \\
\text { miol }\end{array}$ & - & - & - & - & - & 1 & - & - & - & - & - & 1 \\
\hline $\begin{array}{l}\text { Rev. Bras. Med. } \\
\text { Trab. }\end{array}$ & - & - & - & - & - & - & - & - & 1 & - & - & 1 \\
\hline $\begin{array}{l}\text { Rev. Ciên. Saúde } \\
\text { Coletiva }\end{array}$ & 1 & - & - & - & - & - & - & - & - & - & - & 1 \\
\hline $\begin{array}{l}\text { Esc. Anna Nery } \\
\text { Rev. Enferm. }\end{array}$ & - & - & - & - & - & - & - & - & 1 & - & - & 1 \\
\hline $\begin{array}{l}\text { Rev. Latino. En- } \\
\text { ferm. }\end{array}$ & - & - & - & - & - & - & - & - & 1 & - & - & 1 \\
\hline Total & 2 & - & - & - & - & 4 & 1 & - & 4 & 1 & - & 12 \\
\hline
\end{tabular}


Quadro 1. Dados bibliométricos dos artigos selecionados para revisão sistematizada, Brasil, 2020.

\begin{tabular}{|c|c|c|}
\hline TÍTULO DO ARTIGO & ANO & BASE DE VIRTUAL \\
\hline $\begin{array}{l}1 \text { Fatores psicossociais e prevalência da síndrome de burnout entre trabalha- } \\
\text { dores de enfermagem intensivistas. }\end{array}$ & 2015 & Scielo \\
\hline $\begin{array}{l}2 \text { Motivos de afastamentos por licença de saúde dos trabalhadores de enfer- } \\
\text { magem. }\end{array}$ & 2010 & Lilacs \\
\hline $\begin{array}{l}3 \text { Afastamentos do trabalho por transtornos mentais e estressores psicosso- } \\
\text { ciais ocupacionais. }\end{array}$ & 2015 & Lilacs \\
\hline $\begin{array}{l}4 \text { Absenteísmo por transtornos mentais em trabalhadores de saúde em um } \\
\text { hospital no sul do Brasil. }\end{array}$ & 2016 & Scielo \\
\hline $\begin{array}{l}5 \text { As novas relações de trabalho, o desgaste mental do trabalhador e os trans- } \\
\text { tornos mentais no trabalho precarizado. }\end{array}$ & 2010 & Scielo \\
\hline $\begin{array}{l}6 \text { Manifestações de sofrimento: dilemas e desafios para a vigilância em saú- } \\
\text { de do trabalhador. }\end{array}$ & 2015 & Scielo \\
\hline $\begin{array}{l}7 \text { As interações entre os atores no retorno ao trabalho após afastamentos por } \\
\text { transtornos mentais: uma metaetnografia. }\end{array}$ & 2015 & Scielo \\
\hline $\begin{array}{l}8 \text { Transtornos mentais associados ao trabalho em profissionais de enferma- } \\
\text { gem: uma revisão integrativa brasileira }\end{array}$ & 2018 & Lilacs \\
\hline $\begin{array}{l}9 \text { Sintomas depressivos e uso de drogas entre profissionais da equipe de en- } \\
\text { fermagem }\end{array}$ & 2018 & BDenf \\
\hline $\begin{array}{l}10 \text { Aspectos psicossociais do trabalho e distúrbios psíquicos menores na en- } \\
\text { fermagem: uso de modelos combinados }\end{array}$ & 2018 & Lilacs \\
\hline 11 Adoecimento de trabalhadores de enfermagem no contexto hospitalar & 2018 & BDenf \\
\hline $\begin{array}{l}12 \text { Impactos da síndrome de burnout na qualidade de vida dos profissionais } \\
\text { de enfermagem da atenção básica à saúde }\end{array}$ & 2019 & Lilacs \\
\hline
\end{tabular}

Fonte: Pesquisa dos autores

Quadro 2. Publicações selecionadas para discussão, capturadas nas bases Scielo, Lilacs e BDenf publicadas nos últimos 10 anos.

\begin{tabular}{|c|c|c|c|c|}
\hline $\begin{array}{c}\text { AUTOR; } \\
\text { ANO; PAÍS }\end{array}$ & $\begin{array}{l}\text { OBJETIVO DA } \\
\text { PESQUISA }\end{array}$ & $\begin{array}{c}\text { MÉTODO; } \\
\text { TAMANHO DA } \\
\text { AMOSTRA; } \\
\text { TIPO DE ESTUDO }\end{array}$ & $\begin{array}{l}\text { PRINCIPAIS } \\
\text { ACHADOS }\end{array}$ & $\begin{array}{c}\text { CONCLUSÃO DO } \\
\text { ARTIGO }\end{array}$ \\
\hline $\begin{array}{l}\text { Santana LL, } \\
\text { Sarquis LMM, } \\
\text { Brey C, Miran- } \\
\text { da FMD, Felli, } \\
\text { VEA. 2016, } \\
\text { Brasil. }\end{array}$ & $\begin{array}{l}\text { Descrever o perfil } \\
\text { de adoecimento por } \\
\text { transtornos mentais e } \\
\text { comportamentais em } \\
\text { trabalhadores de saúde } \\
\text { de um hospital de ensi- } \\
\text { no no sul do Brasil. }\end{array}$ & $\begin{array}{l}\text { Pesquisa Quantitativa. } \\
\text { Com } 55 \text { registros de } \\
\text { trabalhadores com } \\
\text { CID F do Hospital do } \\
\text { Trabalhador. } \\
\text { Estudo Epidemiológi- } \\
\text { co do tipo transversal } \\
\text { e retrospectivo. }\end{array}$ & $\begin{array}{l}\text { Alto índice de afastamentos por } \\
\text { transtornos mentais e compor- } \\
\text { tamentais em trabalhadores de } \\
\text { saúde, os profissionais mais } \\
\text { afastados foram os técnicos } \\
\text { de enfermagem }(29,09 \%) \text {. As } \\
\text { unidades de terapia intensiva } \\
\text { representaram os setores com } \\
\text { o maior número de dias de ab- } \\
\text { senteísmo totalizando } 81 \% \text { e os } \\
\text { episódios depressivos obtive- } \\
\text { ram a frequência mais signifi- } \\
\text { cativa } 52,72 \% \text { dos transtornos } \\
\text { mentais. }\end{array}$ & $\begin{array}{l}\text { A maioria dos TM é passível de pre- } \\
\text { venção no ambiente de trabalho, des- } \\
\text { de que sua origem seja reconhecida } \\
\text { tanto pelos trabalhadores como pelos } \\
\text { gestores. A partir do reconhecimento } \\
\text { da relação entre as condições de tra- } \\
\text { balho e a ocorrência dos transtornos } \\
\text { será possível realizar as intervenções } \\
\text { necessárias para a prevenção ou re- } \\
\text { dução de tais agravos. }\end{array}$ \\
\hline
\end{tabular}


Quadro 2 (cont.). Publicações selecionadas para discussão, capturadas nas bases Scielo, Lilacs e BDenf publicadas nos últimos 10 anos.

\begin{tabular}{|c|c|c|c|c|}
\hline $\begin{array}{c}\text { AUTOR; } \\
\text { ANO; PAÍS }\end{array}$ & $\begin{array}{l}\text { OBJETIVO DA } \\
\text { PESQUISA }\end{array}$ & $\begin{array}{c}\text { MÉTODO; } \\
\text { TAMANHO DA } \\
\text { AMOSTRA; } \\
\text { TIPO DE ESTUDO } \\
\end{array}$ & $\begin{array}{c}\text { PRINCIPAIS } \\
\text { ACHADOS }\end{array}$ & $\begin{array}{c}\text { CONCLUSÃO DO } \\
\text { ARTIGO }\end{array}$ \\
\hline $\begin{array}{l}\text { Franco T, Dru- } \\
\text { ck G, Silva ES. } \\
\text { 2010, Brasil. }\end{array}$ & $\begin{array}{l}\text { Analisar de que forma } \\
\text { a saúde mental é pre- } \\
\text { judicada pelas atuais } \\
\text { contradições entre mo- } \\
\text { dernização e expansão } \\
\text { de precarização social } \\
\text { e do trabalho. }\end{array}$ & $\begin{array}{l}\text { Análise Crítica. } \\
\text { Ensaio. }\end{array}$ & $\begin{array}{l}\text { Precarização - processo multi- } \\
\text { dimensional (gestão do medo, } \\
\text { práticas participativas forçadas, } \\
\text { multifuncionalidade, seques- } \\
\text { tro de tempo e de criatividade) } \\
\text { que altera a vida dentro e fora } \\
\text { do trabalho. Relações de tra- } \\
\text { balho onde ocorram competi- } \\
\text { ção desenfreada, insegurança } \\
\text { e instabilidade potencializam } \\
\text { a multiexposição aos agentes } \\
\text { físicos, biológicos, químicos e } \\
\text { ergonômicos favorecendo o so- } \\
\text { frimento e o adoecimento dos } \\
\text { trabalhadores. }\end{array}$ & $\begin{array}{l}\text { Refletir sobre os três binômios: 1) } \\
\text { Trabalho e adoecimento; 2) Traba- } \\
\text { lho e degradação/crise ambiental; 3) } \\
\text { Trabalho e precarização social. Prá- } \\
\text { ticas de enfrentamento da precariza- } \\
\text { ção social do trabalho são louváveis, } \\
\text { precisam ser fortalecidas e são uma } \\
\text { necessidade em defesa da vida. Os } \\
\text { adoecimentos e acidentes de trabalho } \\
\text { também são evitáveis. É necessário } \\
\text { resgatar a dignidade no trabalho e sua } \\
\text { função social, impedindo a terceiriza- } \\
\text { ção - ilícita, ilegal e anti social. É ne- } \\
\text { cessário enfrentar a precarização do } \\
\text { mundo do trabalho com expansão do } \\
\text { emprego, favorecendo real inclusão } \\
\text { e pertencimento social (e não apenas } \\
\text { vias compensatórias). A redução da } \\
\text { jornada de trabalho pode favorecer } \\
\text { esta inclusão, sendo, na verdade, um } \\
\text { poderoso instrumento para a geração } \\
\text { de emprego e para as políticas de saú- } \\
\text { de pública. }\end{array}$ \\
\hline $\begin{array}{l}\text { Leão, LHC, } \\
\text { Brandt, LC. } \\
\text { 2015, Brasil. }\end{array}$ & $\begin{array}{l}\text { Realizar uma análise } \\
\text { crítica dos desafios } \\
\text { e dilemas relativos à } \\
\text { integração da saúde } \\
\text { mental no escopo da } \\
\text { vigilância em saúde do } \\
\text { trabalhador no SUS. }\end{array}$ & Análise Crítica. & $\begin{array}{l}\text { Riscos e perigos da tendên- } \\
\text { cia hegemônica centrada na } \\
\text { abordagem epidemiológica e } \\
\text { psicopatologizante. A manifes- } \\
\text { tação do sofrimento deve ser } \\
\text { o foco. Assim, a questão não é } \\
\text { diagnosticar e classificar pes- } \\
\text { soas pelo olhar técnico e mé- } \\
\text { dico, mas a necessária escuta } \\
\text { do sofrimento pelas equipes de } \\
\text { saúde, tendo como referência o } \\
\text { conjunto de situações e contex- } \\
\text { tos que forjam os processos de } \\
\text { subjetivação. }\end{array}$ & $\begin{array}{l}\text { Faz-se necessário enfrentar o desafio } \\
\text { de compreender os fenômenos em } \\
\text { saúde mental e de lidar com situações } \\
\text { complexas, dadas as múltiplas mani- } \\
\text { festações do sofrimento relacionado } \\
\text { ao trabalho e suas diferenças e níveis. } \\
\text { Passos podem ser dados à medida que } \\
\text { investimentos sejam feitos visando à } \\
\text { passagem do modelo mental à produ- } \\
\text { ção das subjetividades, das patologias } \\
\text { às manifestações de sofrimento, do } \\
\text { dado epidemiológico às múltiplas in- } \\
\text { formações, do diagnóstico clínico ao } \\
\text { social, da escuta individual à grupal/ } \\
\text { coletiva, da clínica à heterogeneidade } \\
\text { das intervenções. }\end{array}$ \\
\hline $\begin{array}{l}\text { Neves RF, Nu- } \\
\text { nes MO, Maga- } \\
\text { lhães L. 2015, } \\
\text { Brasil. }\end{array}$ & $\begin{array}{l}\text { Visa: explorar as inte- } \\
\text { rações entre os atores } \\
\text { sociais envolvidos no } \\
\text { retorno ao trabalho; } \\
\text { identificar facilitado- } \\
\text { res ou obstáculos para } \\
\text { o retorno ao trabalho. }\end{array}$ & $\begin{array}{l}\text { Metassíntese Quanti- } \\
\text { tativa. } \\
\text { Estudo Qualitativo. }\end{array}$ & $\begin{array}{l}\text { Com base nas questões da in- } \\
\text { vestigação chegou-se a seis } \\
\text { conceitos de segunda ordem } \\
\text { que se seguem: 1) A experiên- } \\
\text { cia relacionada ao desempenho } \\
\text { do trabalhador no processo de } \\
\text { retorno ao trabalho; 2) O im- } \\
\text { pacto da relação com os cole- } \\
\text { gas no retorno ao trabalho; 3) } \\
\text { Da percepção à ação: o modus } \\
\text { operandi do supervisor no re- } \\
\text { torno ao trabalho; 4) O papel } \\
\text { sinérgico ou antagônico dos } \\
\text { níveis gerenciais; 5) O suporte } \\
\text { dos profissionais de saúde ex- } \\
\text { trapola o ambiente de trabalho; } \\
\text { 6) O papel do mediador no re- } \\
\text { torno ao trabalho. }\end{array}$ & $\begin{array}{l}\text { Os estudos analisados evidenciam } \\
\text { que as expectativas de desempenho } \\
\text { ocupam um lugar central nas relações } \\
\text { entre os atores no contexto do retorno } \\
\text { ao trabalho e podem denunciar a for- } \\
\text { ma como construções sociais sobre o } \\
\text { desempenho no trabalho configuram } \\
\text { um escopo mais amplo sobre o qual } \\
\text { devemos conceber o retorno ao tra- } \\
\text { balho. } \\
\text { O retorno ao trabalho precisa ser en- } \\
\text { tendido numa perspectiva reabilita- } \\
\text { dora, que não ignore os avanços que } \\
\text { as abordagens biomédica e ecológica } \\
\text { trouxeram, mas que avance para um } \\
\text { retorno ao trabalho que inclua novos } \\
\text { modos de viver e trabalhar e que in- } \\
\text { corporem uma perspectiva crítica. }\end{array}$ \\
\hline
\end{tabular}


Quadro 2 (cont.). Publicações selecionadas para discussão, capturadas nas bases Scielo, Lilacs e BDenf publicadas nos últimos 10 anos.

\begin{tabular}{|c|c|c|c|c|}
\hline $\begin{array}{c}\text { AUTOR; } \\
\text { ANO; PAIIS }\end{array}$ & $\begin{array}{l}\text { OBJETIVO DA } \\
\text { PESQUISA }\end{array}$ & $\begin{array}{c}\text { MÉTODO; } \\
\text { TAMANHO DA } \\
\text { AMOSTRA; } \\
\text { TIPO DE ESTUDO }\end{array}$ & $\begin{array}{l}\text { PRINCIPAIS } \\
\text { ACHADOS }\end{array}$ & $\begin{array}{c}\text { CONCLUSÃO DO } \\
\text { ARTIGO }\end{array}$ \\
\hline $\begin{array}{l}\text { Carvalho LSF, } \\
\text { Matos RCS, } \\
\text { Souza NVDO, } \\
\text { Ferreira REDS. } \\
2010 \text {, Brasil. }\end{array}$ & $\begin{array}{l}\text { Identificar o quantita- } \\
\text { tivo de trabalhadores } \\
\text { de enfermagem afas- } \\
\text { tados por licença de } \\
\text { saúde num período de } \\
\text { doze meses e levantar } \\
\text { as causas dessas licen- } \\
\text { ças. }\end{array}$ & $\begin{array}{l}\text { Método de análise } \\
\text { estatística descritiva } \\
\text { simples. } \\
\text { Amostra: } 360 \text { prontu- } \\
\text { ários de trabalhadores } \\
\text { de enfermagem. } \\
\text { Estudo exploratório } \\
\text { descritivo e documen- } \\
\text { tal com abordagem } \\
\text { quantitativa. }\end{array}$ & $\begin{array}{l}\text { Os resultados mostraram que, } \\
\text { no período de janeiro a de- } \\
\text { zembro de } 2007,336 \text { traba- } \\
\text { lhadores de enfermagem ob- } \\
\text { tiveram licença de saúde, dos } \\
\text { quais } 81,85 \% \text { eram auxiliares } \\
\text { de enfermagem. Os motivos } \\
\text { de afastamento foram doenças } \\
\text { osteomusculares, transtornos } \\
\text { mentais e comportamentais. }\end{array}$ & $\begin{array}{l}\text { Concluiu-se que o quadro de adoe- } \\
\text { cimento do coletivo de enfermagem } \\
\text { é alarmante, por conseguinte urge } \\
\text { modificá-lo, através de medidas de } \\
\text { promoção e prevenção de agravos e } \\
\text { riscos à saúde dos trabalhadores. }\end{array}$ \\
\hline $\begin{array}{l}\text { Silva-Junior JS, } \\
\text { Fischer FM. } \\
\text { 2015, Brasil. }\end{array}$ & $\begin{array}{l}\text { Avaliar os fatores asso- } \\
\text { ciados ao afastamento } \\
\text { do trabalho por trans- } \\
\text { tornos mentais relacio- } \\
\text { nados ao trabalho, em } \\
\text { especial a percepção } \\
\text { dos trabalhadores so- } \\
\text { bre fatores psicosso- } \\
\text { ciais no trabalho. }\end{array}$ & $\begin{array}{l}\text { Estudo transversal } \\
\text { analítico realizado em } \\
\text { São Paulo com } 131 \\
\text { requerentes de auxílio- } \\
\text { doença por transtornos } \\
\text { mentais. }\end{array}$ & $\begin{array}{l}\text { Os quadros mais frequentes } \\
\text { foram transtornos depressi- } \\
\text { vos (40,4\%). Entre todos os } \\
\text { requerimentos, } 23,7 \% \text { foram } \\
\text { considerados relacionados ao } \\
\text { trabalho. } \\
\text { O perfil da maioria dos par- } \\
\text { ticipantes era: sexo feminino } \\
(68,7 \%) \text {, até } 40 \text { anos de idade } \\
(73,3 \%) \text {, casado/ união estável } \\
(51,1 \%) \text {, escolaridade igual ou } \\
\text { superior a } 11 \text { anos ( } 80,2 \%) \text {, } \\
\text { não tabagista (80,9\%), não in- } \\
\text { geria bebida alcoólica ( } 84 \%) \text {, } \\
\text { fazia atividade física ( } 77,9 \%) \text {. } \\
\text { Sobre os fatores psicossociais, } \\
\text { prevaleceu trabalho de alta exi- } \\
\text { gência (56,5\%), baixo apoio } \\
\text { social (52,7\%), desequilíbrio } \\
\text { esforço-recompensa (55,7\%) } \\
\text { e comprometimento excessivo } \\
\text { (87,0\%). Não houve associação } \\
\text { estatística entre casos de trans- } \\
\text { tornos mentais relacionados ao } \\
\text { trabalho e as variáveis indepen- } \\
\text { dentes. }\end{array}$ & $\begin{array}{l}\text { A concessão do benefício auxílio-do- } \\
\text { ença acidentário não foi associada a } \\
\text { variáveis sóciodemográficas, hábitos/ } \\
\text { estilo de vida ou fatores psicossociais } \\
\text { no trabalho. A exposição ocupacio- } \\
\text { nal a estressores psicossociais esteve } \\
\text { presente no relato da maioria dos tra- } \\
\text { balhadores afastados do trabalho por } \\
\text { transtornos mentais. Entretanto, di- } \\
\text { versos casos não foram reconhecidos } \\
\text { pela perícia médica previdenciária } \\
\text { como relacionados ao trabalho, o que } \\
\text { pode ter influenciado nos resultados } \\
\text { das associações. }\end{array}$ \\
\hline $\begin{array}{l}\text { JunqueiraMAB, } \\
\text { Santos MA, } \\
\text { Araújo, Ferreira } \\
\text { MCM, Giuliani } \\
\text { CD, Pillon SC. } \\
\text { 2018, Brasil }\end{array}$ & $\begin{array}{l}\text { Avaliar possíveis rela- } \\
\text { ções entre o abuso de } \\
\text { drogas, sintomas su- } \\
\text { gestivos de depressão } \\
\text { e gendero em profis- } \\
\text { sionais da equipe de } \\
\text { enfermagem. }\end{array}$ & $\begin{array}{l}\text { Estudo com aborda- } \\
\text { gem transversal reali- } \\
\text { zado em hospital geral, } \\
\text { com } 416 \text { participantes. } \\
\text { Usado questionário } \\
\text { com informações so- } \\
\text { ciodemográfica, os } \\
\text { testes ASSIST, AU- } \\
\text { DIT-C e PHQ-2. Usa- } \\
\text { dos os Testes Exato de } \\
\text { Fisher, Teste de Qui- } \\
\text { Quadrado, e Análise } \\
\text { de Regressão Logísti- } \\
\text { ca Multivariada }\end{array}$ & $\begin{array}{l}\text { Gênero feminino apresentaram } \\
\text { razões de chances diminuídas } \\
\text { para o consumo de álcool no } \\
\text { padrão binge e uso de maco- } \\
\text { nha; e razões de chances dobra- } \\
\text { das para sentimentos de falta } \\
\text { de interesse e prazer. Sintomas } \\
\text { sugestivos de depressão apre- } \\
\text { sentaram riscos potenciais para } \\
\text { o uso de sedativos. }\end{array}$ & $\begin{array}{l}\text { Uso abusivo de drogas e sintomas de } \\
\text { depressão estão associados e mani- } \\
\text { festam-se diferentemente, de acordo } \\
\text { com o gênero entre os profissionais } \\
\text { da equipe de enfermagem }\end{array}$ \\
\hline
\end{tabular}


Quadro 2 (cont.). Publicações selecionadas para discussão, capturadas nas bases Scielo, Lilacs e BDenf publicadas nos últimos 10 anos.

\begin{tabular}{|c|c|c|c|c|}
\hline $\begin{array}{c}\text { AUTOR; } \\
\text { ANO; PAIÍS }\end{array}$ & $\begin{array}{l}\text { OBJETIVO DA } \\
\text { PESQUISA }\end{array}$ & $\begin{array}{c}\text { MÉTODO; } \\
\text { TAMANHO DA } \\
\text { AMOSTRA; } \\
\text { TIPO DE ESTUDO }\end{array}$ & $\begin{array}{l}\text { PRINCIPAIS } \\
\text { ACHADOS }\end{array}$ & $\begin{array}{c}\text { CONCLUSÃO DO } \\
\text { ARTIGO }\end{array}$ \\
\hline $\begin{array}{l}\text { Fernandes MA, } \\
\text { Soares LMD, } \\
\text { Soares Silva J. } \\
\text { 2018, Brasil. }\end{array}$ & $\begin{array}{l}\text { Levantar estudos bra- } \\
\text { sileiros com vistas a } \\
\text { identificar a relação } \\
\text { causal entre os trans- } \\
\text { tornos mentais e o tra- } \\
\text { balho dos profissionais } \\
\text { da enfermagem. }\end{array}$ & $\begin{array}{l}\text { Revisão integrativa } \\
\text { de estudos brasileiros } \\
\text { do período de } 2010 \text { a } \\
\text { 2017. Para o levanta- } \\
\text { mento bibliográfico } \\
\text { foi utilizado o banco } \\
\text { de dados da Biblioteca } \\
\text { Virtual em Saúde. }\end{array}$ & $\begin{array}{l}\text { Descobriu-se que o sofrimento } \\
\text { psíquico prejudica a vida fami- } \\
\text { liar, social, pessoal, laboral, os } \\
\text { estudos, a compreensão de si } \\
\text { mesmo e dos outros, a capaci- } \\
\text { dade de autocrítica, a aceitação } \\
\text { dos problemas e a possibilidade } \\
\text { de ter prazer na vida em geral. } \\
\text { A carga física através de grande } \\
\text { volume de atividades, déficit de } \\
\text { pessoal e número elevado de } \\
\text { pacientes, bem como a pressão } \\
\text { psicológica sobre o trabalho, } \\
\text { contribuem para o surgimento } \\
\text { de transtornos mentais. }\end{array}$ & $\begin{array}{l}\text { As pressões sofridas pelo trabalhador } \\
\text { da enfermagem no ambiente laboral } \\
\text { e as jornadas duplas de trabalho, as- } \\
\text { sociadas ao modo de enfrentamento } \\
\text { do trabalhador e à baixa remuneração } \\
\text { contribuem para o afastamento por } \\
\text { transtornos mentais. Entretanto, se } \\
\text { faz necessária a realização de estudos } \\
\text { mais amplos. }\end{array}$ \\
\hline $\begin{array}{l}\text { Baptista ATP, } \\
\text { Souza NVDO, } \\
\text { G a } 11 \text { a s c h i } \\
\text { CH, Varella } \\
\text { TCMML, No- } \\
\text { ronha IR. 2018 }\end{array}$ & $\begin{array}{l}\text { Determinar as questões } \\
\text { de saúde que levam os } \\
\text { profissionais de enfer- } \\
\text { magem a licenciarem- } \\
\text { se do trabalho por mais } \\
\text { de } 15 \text { dias ou sofrerem } \\
\text { readaptação funcional }\end{array}$ & $\begin{array}{l}\text { Pesquisa documen- } \\
\text { tal, retrospectiva com } \\
\text { abordagem quantitati- } \\
\text { va. Foram analisados } \\
886 \text { registros de afas- } \\
\text { tamentos da equipe } \\
\text { de enfermagem de um } \\
\text { hospital universitário. } \\
\text { Os dados foram apre- } \\
\text { sentados por meio de } \\
\text { estatística descritiva. }\end{array}$ & $\begin{array}{l}\text { Os afastamentos dos trabalha- } \\
\text { dores foram atribuídos, princi- } \\
\text { palmente, transtornos mentais } \\
\text { e comportamentais (22,6\%), } \\
\text { seguidos das doenças do siste- } \\
\text { ma osteomuscular e do tecido } \\
\text { conjuntivo }(24,4 \%) \text { dos casos. } \\
\text { Em relação aos motivos que } \\
\text { levaram os profissionais a so- } \\
\text { frerem readaptação funcional, } \\
\text { captou-se como principal cau- } \\
\text { sa as doenças osteomusculares } \\
(56,3 \%) \text {, registrando-se em } \\
\text { seguida os transtornos mentais } \\
(18,8 \%) \text {. }\end{array}$ & $\begin{array}{l}\text { Os trabalhadores possuem elevado } \\
\text { índice de adoecimento por doenças } \\
\text { osteomusculares, no entanto, preocu- } \\
\text { pa o crescente adoecimento mental. } \\
\text { Portanto, é necessário intensificar } \\
\text { medidas protetoras para prevenção } \\
\text { do adoecimento e melhoradas condi- } \\
\text { ções laborais. }\end{array}$ \\
\hline $\begin{array}{lr}\text { Pinhatti } & \text { EDG, } \\
\text { Ribeiro } & R P, \\
\text { Soares } & M H, \\
\text { Martins } & J T, \\
\text { Lacerda } & \text { MR, } \\
\text { Galdino } & \text { MJQ. } \\
\text { 2018, Brasil. }\end{array}$ & $\begin{array}{l}\text { Analisar o uso de } \\
\text { modelos combinados } \\
\text { para a avaliação dos } \\
\text { aspectos psicossociais } \\
\text { no trabalho e sua as- } \\
\text { sociação com a pre- } \\
\text { valência de Distúrbios } \\
\text { Psíquicos Menores } \\
\text { entre trabalhadores da } \\
\text { enfermagem. }\end{array}$ & $\begin{array}{l}\text { Estudo transversal rea- } \\
\text { lizado com uma amos- } \\
\text { tra de } 285 \text { trabalhado- } \\
\text { res de enfermagem. } \\
\text { A coleta de dados foi } \\
\text { realizada por meio da } \\
\text { aplicação de questio- } \\
\text { nário estruturado con- } \\
\text { tendo características } \\
\text { sociodemográficas, } \\
\text { ocupacionais e os ins- } \\
\text { trumentos Demand } \\
\text { Control } \\
\text { Support, Effort- } \\
\text { Reward Imbalance e } \\
\text { Self-Reporting Ques- } \\
\text { tionnaire. Realizou- } \\
\text { se análise descritiva } \\
\text { e regressão logística } \\
\text { múltipla. }\end{array}$ & $\begin{array}{l}\text { A prevalência de suspeição de } \\
\text { Distúrbios Psíquicos Menores } \\
\text { foi de } 32,6 \% \text {. As dimensões de } \\
\text { ambos os modelos associaram- } \\
\text { se à saúde mental. Os modelos } \\
\text { completos Desequilíbrio Esfor- } \\
\text { ço-Recompensa e Demanda- } \\
\text { Controle e Suporte Social pre- } \\
\text { veem os Distúrbios Psíquicos } \\
\text { Menores em maior grau do que } \\
\text { o uso combinado dos modelos } \\
\text { parciais. }\end{array}$ & $\begin{array}{l}\text { Constatou-se que o modelo Desequi- } \\
\text { líbrio Esforço-Recompensa captou } \\
\text { melhor a magnitude dos Distúrbios } \\
\text { Psíquicos Menores nessa amostra } \\
\text { de trabalhadores em comparação ao } \\
\text { Demanda-Controle e Suporte social. } \\
\text { Contudo, o uso de ambos os modelos } \\
\text { teóricos revelou contribuições sin- } \\
\text { gulares na avaliação dos Distúrbios } \\
\text { Psíquicos Menores e considerando a } \\
\text { complexidade do adoecimento men- } \\
\text { tal é importante que diferentes fato- } \\
\text { res sejam avaliados. }\end{array}$ \\
\hline $\begin{array}{l}\text { Ramos CEB, } \\
\text { Farias JÁ, Cos- } \\
\text { ta MBS, Fonse- } \\
\text { ca, LCT. 2019, } \\
\text { Brasil. }\end{array}$ & $\begin{array}{l}\text { Identificar o impacto } \\
\text { da Síndrome de Bur- } \\
\text { nout (SB) na qualidade } \\
\text { de vida dos profissio- } \\
\text { nais de enfermagem } \\
\text { da Atenção Básica à } \\
\text { Saúde. }\end{array}$ & $\begin{array}{l}\text { Estudo exploratório e } \\
\text { descritivo, com cará- } \\
\text { ter quanti-qualitativo, } \\
\text { desenvolvido com } 52 \\
\text { profissionais da Rede } \\
\text { de Atenção Básica à } \\
\text { Saúde da Cidade de } \\
\text { Bayeux, região me- } \\
\text { tropolitana de João } \\
\text { Pessoa-PB. }\end{array}$ & $\begin{array}{l}13,5 \% \text { das profissionais estuda- } \\
\text { das manifestaram característi- } \\
\text { cas relacionadas à SB e } 30,8 \% \\
\text { apresentaram predisposição } \\
\text { para o desenvolvimento da } \\
\text { mesma. }\end{array}$ & $\begin{array}{l}\text { O profissional acometido pela SB } \\
\text { tende a apresentar diminuição em seu } \\
\text { rendimento de trabalho e dificuldades } \\
\text { na relação com a equipe de trabalho, } \\
\text { refletindo o impacto negativo que a } \\
\text { síndrome traz para a qualidade de } \\
\text { vida. }\end{array}$ \\
\hline
\end{tabular}

Fonte: Pesquisa dos autores 
resultados e conclusões dos artigos selecionados, encontra-se apresentada no quadro 2 .

\section{Discussão}

Dentre os estudos analisados pode-se observar que a maioria dos afastamentos se dá entre as mulheres, visto que a maior parcela dos profissionais de enfermagem é do sexo feminino. A categoria profissional mais afetada por transtornos ansiosos foi a de nível médio.

O perfil de adoecimento de trabalhadores por transtorno mental evidencia a relação entre as condições de trabalho e a ocorrência dos mesmos ${ }^{6,7}$. Os transtornos mentais mais frequentes entre os trabalhadores foram os episódios depressivos (CID-10 F32) com 40\%, seguidos dos outros transtornos ansiosos (CID-10 F41) $\operatorname{com} 20 \%{ }^{2}$.

Os transtornos mentais acarretam grande impacto sobre os indivíduos, as famílias e as comunidades, em face da incapacitação para atividades ocupacionais, lazer e muitas vezes do convívio social. No caso dos transtornos depressivos, ocupam o quarto lugar no mundo, dentre todas as outras doenças incapacitantes. De acordo com dados Organização Mundial de Saúde (OMS) se persistirem as tendências atuais, a depressão será a segunda maior causa de disfunção em todo o mundo ${ }^{8}$.

A rotina de trabalho da equipe de enfermagem carrega vários elementos estressores tais como: alta carga psíquica; sobrecarga de atividades; fatores organizacionais; dentre outros que ocasionam consequências no exercício profissional. A equipe de enfermagem deve ter boas condições físicas e mentais para prestar o cuidado adequado, mas para isso deve receber atenção especial para que o desempenho de suas atividades ocorra sem prejuízo à sua saúde 9 .

A mulher tem seu potencial de risco aumentado, ao assumir duplas ou triplas jornadas de trabalho, para conseguir manter suas responsabilidades considerando tanto as exigidas pela sociedade como pela família ${ }^{10,11}$.

A precarização do trabalho pontua que os adoecimentos e acidentes de trabalho são evitáveis, a legislação do trabalho deve ser preservada e cumprida, para que o mundo do trabalho consiga alcançar níveis civilizados $^{12,13,14}$.

Os profissionais de enfermagem são expostos diariamente a fatores estressores em seu local de trabalho, independentemente se a assistência prestada é junto ao leito ou na área administrativa. Essa categoria profissional apresenta doenças mentais ou está susceptível as mesmas. O rendimento de trabalho também é comprometido e dificuldades na relação com a equipe de trabalho é notada ${ }^{15,16}$.

Em 2020, com a pandemia da Covid-19, o mundo volta seus olhares para a saúde mental dos seus trabalhadores que estão na linha de frente. Pois, não se pode minimizar as repercussões psicológicas que o cenário gerado pela pandemia possa causar sobre os profissionais de saúde. É sabido, que para o enfrentamento e contenção de um surto pandêmico, se faz necessário garantir, assistência apropriada em saúde mental, envolvendo ações voltadas aqueles com sofrimento mental ao longo da crise ${ }^{17}$.

\section{Considerações finais}

Observou-se que há necessidade de se realizar mais estudos para a produção de informação para embasar medidas de prevenção, e enfrentamento dos transtornos mentais entre os trabalhadores de saúde. A saúde mental, o sofrimento e trabalho ocupam cada vez mais a realidade do trabalhador de enfermagem, e vai muito além de diagnosticar e notificar os transtornos relatados por esses profissionais. É fundamental que os profissionais entendam que a necessidade do autocuidado, e de minimizar situações que levem ao seu esgotamento e provável adoecimento.

Os investimentos sobre essa temática devem ser realizados, visando à educação permanente dos trabalhadores de enfermagem e dos gestores, os quais devem estar cientes que o campo da saúde do trabalhador deve estar em constante mudança, para construção de assistência segura e de qualidade. O enfermeiro deve estar atento às subjetividades do trabalhador, observando as facilidades e dificuldades enfrentadas, durante o processo de trabalho a fim de construir mudança na prática onde os trabalhadores se beneficiem com as estratégias planejadas.

A prevenção dos transtornos mentais deve ser realizada, por meio de entrevistas de mapeamento que revelem a subjetividade do trabalhador, a fim de que seja compreendido e consiga realizar da melhor maneira possível o seu trabalho. O ambiente laboral deve ser criteriosamente avaliado para que indicadores possam indicar grupos de riscos a serem trabalhados com campanhas de informação e educativas, para controle e eliminação dos fatores de risco, bem como para proteção dos trabalhadores evitando a depressão e distúrbios de ansiedade.

\section{Referências}

1. Barcaui A. Estudo da Evolução do Sofrimento. Cadernos de Estudos e Pesquisas - Journal of Studies and Research, 2011;15(34):21-30.

2. Silva-Junior JS, Fischer FM. Afastamentos do trabalho por transtornos mentais e estressores psicossociais ocupacionais. Rev. Bras. Epidemiol. 2015;18(6):735-744.

3. Brasil. Doenças relacionadas ao trabalho: manual de procedimentos para os serviços de saúde. Brasília: Ministério da Saúde do Brasil, 2001:580.

4. Franco T, Druck G, Seligmann-Silva E. As novas relações de trabalho, 
o desgaste mental do trabalhador e os transtornos mentais no trabalho precarizado. Rev. Bras. Saúde Ocup., 2010;35(122):229-248.

5. Silva JLL. Estresse e transtornos mentais comuns em trabalhadores de enfermagem. Rev. Eletr. Enf. 2008;10(4):1174-5.

6. Santana LL. et al. Absenteísmo por transtornos mentais em trabalhadores de saúde em um hospital no sul do Brasil. Rev. Gaúcha Enferm, 2016;37(1):1-8.

7. Silva AG. Depressão. Semana Nacional de Luta e Conscientização Sobre a Depressão - Audiência pública PL1938/15. Asso. Bras. de psiquiatria. CSSF, Brasília. 2017.

8. Carvalho LSF, et al. Motivos de afastamentos por licença de saúde dos trabalhadores de enfermagem. Ciênc. Cuid. Saúde, 2009;9(1):60-66.

9. Ramos CEB, Farias JA, Costa MBS, Fonseca LCT. Impactos da síndrome de burnout na qualidade de vida dos profissionais de enfermagem da atenção básica à saúde. Revista Brasileira de Ciências da Saúde. 2019;23(3):285-296. [Access 14 de maio 2020]; Available in: DOI: https:// doi.org/10.22478/ufpb.2317-6032.2019v23n3.43595.

10. Junqueira MAB, Santos MA, Araújo LB, Ferreira MCM, Giuliani CD, Pillon SC. Sintomas depressivos e uso de drogas entre profissionais da equipe de enfermagem. Esc Anna Nery 2018;22(4):e20180129. [Access 14 de maio 2020]; Available in: DOI 10.1590/2177-9465-EAN-2018-0129.

11. Pinhatti EDG, Ribeiro RP, Soares MH, Martins JT, Lacerda MR, Galdino MJQ. Psychosocial aspects of work and minor psychic disorders in nursing: use of combined models. Rev. Latino-Am. Enfermagem. 2018;26:e3068. [Access 14 de maio 2020]; Available in: DOI: http://dx.doi. org/10.1590/1518-8345.2769.3068.

12. Fernandes MA, et al. Transtornos mentais associados ao trabalho em profissionais de enfermagem. Rev Bras Med Trab. 2018;16(2):218-24. [Access 14 de maio 2020]; Available in: DOI: 10.5327/Z1679443520180228.

13. Baptista ATP, Souza NVDO, Gallasch CH, Varella TCMYML, Noronha IR, Noronha IR. Adoecimento de trabalhadores de enfermagem no contexto hospitalar. Rev enferm UERJ, Rio de Janeiro, 2018; 26:e31170.

14. Silva JLL, et al. Fatores psicossociais e prevalência da síndrome de burnout entre trabalhadores de enfermagem intensivistas. Rev. Bras. Ter. Intensiva. 2015;27(2):125-133. [Access 14 de maio 2020]; Available in: https:/www.scielo.br/pdf/rbti/v27n2/0103-507X-rbti-27-02-0125.pdf.

15. Leão LHC, Brandt LC. Manifestações de sofrimento: dilemas e desafios para a vigilância em saúde do trabalhador. Physis. 2015; 25(4):12711292. Available in: https://www.scielo.br/pdf/physis/v25n4/0103-7331physis-25-04-01271.pdf.

16. Neves RF, Nunes MO, Magalhães L. As interações entre os atores no retorno ao trabalho após afastamentos por transtornos mentais: uma metaetnografia. Cad. Saúde Pública. 2015; 31(11):2275-2290. Available in: https://www.scielo.br/pdf/csp/v31n11/0102-311X-csp-31-11-2275.pdf.

17. Faro A, Bahiano MA, Nakano TC, Reis C, Silva BFP, Vitti LS. COVID-19 and mental health: The emergence of care. Copyright. 2020; [Access 14 de maio 2020]; Available in: DOI: https://doi.org/10.1590/ SciELOPreprints. 146. 